\title{
Delayed Visual Evoked Response is Linked to Vascular Endothelial Growth Factor and Autonomic Dysfunction in Type 2 Diabetes Mellitus
}

\author{
Allampalli Sirisha ${ }^{1}$, Yerrabelli Dhanalakshmi ${ }^{1^{*}}$, Gopal Krushna Pal ${ }^{1}$, Mukta Wyawahare ${ }^{1}$, Shashi Ahuja ${ }^{2}$ \\ ${ }^{1}$ Department of Physiology Medicine, Jawaharlal Institute of Post-graduate Medical Education and Research, Puducherry, India; \\ ${ }^{2}$ Department of Optholmology, Jawaharlal Institute of Post-graduate Medical Education and Research, Puducherry, India
}

\begin{abstract}
Aims: In this study, we aimed to assess the link between visual evoked potentials (VEP), angiogenic marker (VEGF) and autonomic function tests in type-2 diabetes mellitus (T2DM).

Methods: Age-matched 86 male subjects (44 type-2 diabetics on treatment and 42 normal healthy volunteers) were recruited for this study. Body mass index (BMI), basal cardiovascular parameters, spectral analysis of heart rate variability (HRV), were assessed. Fasting blood glucose, insulin, HOMA-IR and VEGF were estimated. The independent association of the P100 latency of VEP with VEGF was determined by multiple regression analysis.

Results: LF-HF ratio a marker of sympathovagal balance, increased significantly $(\mathrm{p}=0.0287$ ) in diabetic group compared to the control group. Comparison of visual evoked potentials between controls and diabetic groups showed significant increase in latency of $\mathrm{P} 100$ of both the eyes (right eye $\mathrm{p}<0.0001$, left eye $\mathrm{p}=0.0003$ ) among the cases. There was significant increase $(\mathrm{p}=0.0091)$ in VEGF in diabetic subjects when compared to control. Also positive and significant correlation of P100 latency of VEP with VEGF is seen in diabetics.

Conclusions: There is delayed latency of VEP and increased VEGF in type 2 diabetic people. The correlation between VEP and VEGF could indicate the probable role of angiogenesis leading to reduced integrity of the neural pathways in T2DM.
\end{abstract}

Keywords: Vascular endothelial growth factor; Visual evoked potentials; Type 2 diabetes mellitus; Autonomic dysfunction

\section{INTRODUCTION}

Diabetes mellitus (DM) is a metabolic disorder, characterized by chronic hyperglycemia together with disturbances of carbohydrate, fat and protein metabolism resulting from defects of insulin secretion, insulin action, or both [1,2]. According to recent ICMR-INDIAB national study there are 366 million people with diabetes globally and 62.4 million people with type 2 diabetes (T2DM) in India [3]. Patients with diabetes have an increased incidence of atherosclerotic cardiovascular, retinal, peripheral arterial and cerebrovascular disease. Perhaps one of the most overlooked of all serious complications of diabetes is cardiac autonomic neuropathy (CAN), which can be diagnosed by non-invasive tests like heart rate variability (HRV), whereas reduced HRV is the earliest indicator of CAN [4-6]. Diabetic retinopathy is the most frequent cause of new cases of blindness among adults aged 20-74 years. During the first two decades of disease, nearly all patients with type 1 diabetes and 60\% of patients with T2DM have retinopathy [7]. The duration of diabetes is probably the strongest predictor for development and progression of retinopathy [7,8]. In diabetes, visual deficit appears from both, vascular disease and metabolic abnormalities, which can affect the retina and visual pathways. Recording of visual evoked potentials (VEP) in response to pattern reversal stimuli is a very sensitive test for detecting these abnormalities by increased latency and decreased amplitude [9]. New vessel formation in diabetic retinopathy causes visual loss with vitreous haemorrhage, retinal detachment, and neovascular glaucoma. Retinal neovascularization has been thought to occur due to the

*Correspondence to: Dr. Yerrabelli Dhanalakshmi, Associate Professor, Department of Physiology Medicine, Jawaharlal Institute of Post-graduate Medical Education and Research, Puducherry, India, 605006; Tel: +91 9444781210; E-mail: beenulakshmi70@gmail.com

Received: August 19, 2018; Accepted: September 18, 2019; Published: September 25, 2019

Citation: Sirisha A, Dhanalakshmi Y, Pal GK, Wyawahare M, Ahuja S (2019) Delayed Visual Evoked Response is Linked to Vascular Endothelial Growth Factor and Autonomic Dysfunction in Type 2 Diabetes Mellitus. J Diabetes Metab 10:833. doi: 10.35248/2155-6156.19.10.833

Copyright: (C) 2019 Sirisha A, et al. This is an open-access article distributed under the terms of the Creative Commons Attribution License, which permits unrestricted use, distribution, and reproduction in any medium, provided the original author and source are credited. 
release of local angiogenic factors by ischaemic and hypoxic retina [10]. Elevated serum vascular endothelial growth factor (VEGF) has drawn much attention relating to the pathogenesis of diabetic retinopathy [11]. Evidences suggest that VEGF, a cytokine that acts as an endothelial cell mitogen that induces increased microvascular permeability is causally involved in the development of diabetic retinopathy [12]. Identification of such vasculogenic triggering factor and detection of an early biologic marker for diabetic retinopathy may help us to device methods to prevent progression of neovascularization in diabetic retinopathy. Although intraocular levels of VEGF have been studied extensively, role of systemic vasculogenic factors in pathophysiology of diabetic retinopathy has not been fully elucidated in previous studies. In particular, there is no report to suggest the association between systemic vasculogenic factors and diabetic retinopathy. To best of our knowledge, the link of visual evoked response with VEGF and autonomic dysfunction is not established till date. Even though, retinopathy development and cardiovascular disease (CVD) in diabetes share common risk factor such as hypertension, dyslipidemia, and elevated HbA1c level the pathophysiology and underlying the association of retinopathy with CVD is not well understood. Therefore, in the present study we have assessed the link between VEP, VEGF and autonomic functions in T2DM.

\section{MATERIALS AND METHODS}

The present study was conducted in the Department of Physiology, Jawaharlal Institute of Postgraduate Medical Education and Research (JIPMER), Pondicherry, India. After obtaining approval of the project plan from research and ethics committees of Institute.

\section{Subjects}

This cross-sectional study was done in two groups:

- Study group: 44 type 2 diabetic patients on treatment with less than ten years of duration.

- Control group: 42 age and gender matched healthy volunteers.

Written informed consent was obtained from all the subjects participated in this study before the initiation of any study procedure.

Exclusion criteria for study group: Individuals with history of smoking and/or alcoholism, acute or chronic ailments, and known cases of hypertension, cardiac and renal disease, or any endocrinal disorder were excluded from the study. Patients with diabetic retinopathy, cataract, glaucoma, macular edema, vitreous opacities or any evidence of optic atrophy were excluded from the study.

Exclusion criteria for control group: Subjects suffering from any illness like diabetes, hypertension, heart disease, autonomic failure, and any other endocrine disorders or on any hormonal therapy or drugs affecting autonomic function, and any visual abnormalities.

Criteria used to diagnose and to exclude T2DM:
- For study group T2DM patients recruited from the diabetic clinic, who are diagnosed with diabetes with minimum 6 months of duration and were on treatment.

- For control group fasting blood glucose and basal blood pressure recording were done and people whose values are within normal range were recruited.

\section{Brief procedure}

Study participants recruited from Diabetic Clinic, were asked to report to the Department of Physiology, at around 8:00 A.M with overnight fasting. They were instructed to avoid coffee, tea, and any drug that influence the autonomic nervous system for $12 \mathrm{~h}$ before the test. They were further instructed to shampoo and dry their hair. The subjects were instructed to avoid any miotic or mydriatic drugs 12 hours before the test. Height and weight were measured to calculate body mass index (BMI). After $10 \mathrm{~min}$ of supine rest in polygraph laboratory (room temperature maintained at $25^{\circ} \mathrm{C}$ ), the following recordings were done.

Recording of baseline cardiovascular (CV) and HRV parameters: Baseline HR and blood pressure (BP) were recorded in the left arm after $10 \mathrm{~min}$ of rest in the supine position using automatic BP monitor (Omron Healthcare Co. Ltd, Kyoto, Japan). For recording of short-term HRV, the procedure as described earlier [13] and recommendation of the Task Force on HRV was followed [14]. For the purpose, ECG signals were digitalized using ML870 Power Lab 8/30 AD Instruments Data Acquisition Systems and stored for offline analysis by computer software-Kubios HRV, version 2.1, Kuopio, Finland. The software detected Rwave and computed all the RR intervals from the ECG recording. Frequency domain indices such as total power (TP), low-frequency power expressed in normalized unit (LFnu), high-frequency power expressed in normalized unit (HFnu), ratio of LF to HF power (LF-HF ratio), and time domain indices (TDI) such as mean RR, square root of the mean squared differences of successive normal to normal intervals (RMSSD), standard deviation of normal to normal interval (SDNN), the number of interval differences of successive NN intervals greater than $50 \mathrm{~ms}$ (NN50), and the proportion derived by dividing NN50 by the total number of $\mathrm{NN}$ intervals (pNN50) were recorded.

Measurement of Visual Evoked Potentials (VEP): VEP were recorded using pattern reversal stimulation [15]. The scalp was prepared by abrading and degreasing. Binocular, pattern-reversal checker board stimulation of $1.8 \mathrm{~Hz}$ frequency was used. The distance between the TV screen and each subject was $120 \mathrm{~cm}$. The patient was instructed to fix his gaze at the centre of the screen. An average of 200 sweeps of stimuli was given to each eye and the visual function was assessed with the help of P100 wave latency. The bioelectrical signals were recorded by silver or silver chloride disc electrodes placed at: 1) Grounding (FPZ), 2) Active (OZ), 3) Reference ( $\mathrm{Fz}$ ) using electrode paste according to 10-20 international system of EEG electrode placement. Uniform illumination was maintained in the laboratory and the electrode impedance was kept below $5 \mathrm{k}$. The evoked responses were aver-aged and analyzed by the Evoked Potential Recorder (Nihon Kohden Electrophysiology/ Electromyography 
instrument). The peak N75, P100 and N145 latencies were recorded.

Measurement of biochemical parameters: Five milliliter of venous blood (with overnight fasting) was collected from both cases and control subjects under aseptic precautions for biochemical analysis of fasting blood glucose (FBG), insulin and VEGF. FBG was assessed using fully automated analyzer (AU400, Olympus). Insulin (Diagnostic kit from DiasourceInc, New York) and VEGF (Diagnostic kit from Orgenium laboratories, Finland) were estimated by using enzyme immunoassay method.

\section{Statistical analysis}

SPSS version 19 was used for statistical analysis. The data were subjected to Kolmogorov-Smirnov normality test. All the data were expressed as mean $\pm \mathrm{SD}$. The intergroup differences in mean between the controls and cases were compared using Student's unpaired ' $t$ '-test, for normally distributed data. The association of VEP with biochemical parameters and autonomic functions was assessed by Pearson's correlation analysis. Multiple regression analysis was carried out to identify the independent factors associated with the delayed latency of VEP. The difference was considered statistically significant if probability of chance was less than 0.05 .

\section{RESULTS}

In type 2 diabetic subjects, the baseline $\mathrm{CV}$ parameters, such as BHR ( $p=0.0079), \operatorname{SBP}(p=0.0001)$, DBP $(p=0.0043)$, and RPP $(p<0.0001)$, were significantly high when compared with age, gender and BMI matched controls (Table 1). In frequency domain HRV parameters, TP and HFnu were significantly less ( $p<0.0001$ and 0.0358) and LFnu and LFHF ratio were significantly high (Table 2), whereas all the time domain indices of HRV such as RMSSD, SDNN, NN50, and pNN50 were significantly decreased $(p<0.0001)$ in type 2 diabetic subjects compared to apparently healthy controls (Table 2). Comparison of VEP between controls and diabetic groups showed significant increase in latency of P100 of both the eyes (Right eye p<0.0001, Left. Eye $\mathrm{p}=0.0003$ ) among the cases. Also, N75-P100 amplitude of both eyes (Right eye $p=0.0222$, Left Eye $p=0.0022$ ) were significantly decreased in the study group compared to controls. There was no significant difference in N75 and N145 latencies (Table 3). Table 4 shows increased FBG, insulin and Homeostasis model of assessment insulin resistance (HOMA IR) are elevated $(p<0.0001)$ in study group along with significant increase in VEGF in comparison to the controls $(p=0.0083)$. The correlation of VEGF with BMI, basal CV parameters, HOMA-IR and VEP parameters shows that there was a significant positive correlation of P100 latency of both the eyes $(\mathrm{r}=0.3603, \mathrm{p}=0.0163$ for right eye and $\mathrm{r}=0.3693, \mathrm{p}=0.0136$ for left eye) with VEGF. No correlation was observed with VEGF to other parameters (Table 5). Tables 6 and 7 shows the multiple regression analysis of P100 latency of VEP for both right and left eye respectively which revealed the independent contribution of $\operatorname{VEGF}(\beta 0.360, \mathrm{p}=0.016$ and $\beta 0.397 \mathrm{p}=0.024)$.
Table 1: Comparison of age and anthropometric parameters and baseline CV parameters between control and diabetic groups.

\begin{tabular}{lccc}
\hline \multicolumn{1}{c}{ Parameter } & $\begin{array}{c}\text { Controls } \\
(\mathbf{n}=42)\end{array}$ & $\begin{array}{c}\text { Diabetics } \\
(\mathbf{n}=44)\end{array}$ & p value \\
\hline Age & $41.5 \pm 9.46$ & $44.27 \pm 8.05$ & 0.1465 \\
\hline Body weight $(\mathrm{kg})$ & $66.67 \pm 10.12$ & $66.25 \pm 11.39$ & 0.8578 \\
\hline BMI $\left(\mathrm{Kg} / \mathrm{m}^{2}\right)$ & $24.01 \pm 3.64$ & $25.86 \pm 5.51$ & 0.0711 \\
\hline BHR $(\mathrm{bpm})$ & $69.47 \pm 10.77$ & $75.97 \pm 11.36$ & 0.0079 \\
\hline SBP $(\mathrm{mmHg})$ & $114.98 \pm 9.15$ & $125.48 \pm 13.70$ & 0.0001 \\
\hline DBP $(\mathrm{mmHg})$ & $70.21 \pm 6.61$ & $76.11 \pm 11.29$ & 0.0043 \\
\hline MAP $(\mathrm{mmHg})$ & $85.13 \pm 6.50$ & $92.56 \pm 11.06$ & 0.0003 \\
\hline RPP $(\mathrm{mmHg} / \mathrm{min})$ & $79.87 \pm 9.85$ & $95.32 \pm 15.57$ & $<0.0001$ \\
\hline
\end{tabular}

Values expressed as mean $\pm \mathrm{SD}$; Analysis done by student's unpaired $\mathrm{t}$ test. The $\mathrm{P}$ values less than 0.05 were considered statistically significant.BMI: Body Mass Index, HR: Heart rate, SBP: Systolic blood pressure, DBP: Diastolic blood pressure; MAP: Mean arterial pressure, RPP: Rate pressure product.

Table 2: Comparison of frequency domain indices of HRV between control and diabetic groups.

\begin{tabular}{lccc}
\hline \multicolumn{1}{c}{ Parameter } & Controls $(\mathbf{n}=42)$ & Diabetics $(\mathbf{n}=44)$ & p value \\
\hline TP & $530.14 \pm 197.95$ & $156.93 \pm 96.00$ & $<0.0001$ \\
\hline LFnu & $50.03 \pm 19.86$ & $58.61 \pm 17.39$ & 0.0358 \\
\hline HFnu & $49.96 \pm 19.86$ & $41.38 \pm 17.39$ & 0.0358 \\
\hline LF-HF ratio & $1.35 \pm 1.04$ & $1.98 \pm 1.51$ & 0.0287 \\
\hline Mean RR (s) & $0.84 \pm 0.13$ & $0.77 \pm 0.11$ & 0.0194 \\
\hline SDNN (ms) & $43.16 \pm 20.89$ & $22.09 \pm 11.02$ & $<0.0001$ \\
\hline RMSSD (ms) & $36.78 \pm 17.2$ & $19.88 \pm 19.88$ & $<0.0001$ \\
\hline NN50 & $54.71 \pm 42.61$ & $16.52 \pm 11.48$ & $<0.0001$ \\
\hline p NN50 & $16.46 \pm 13.51$ & $4.95 \pm 2.28$ & $<0.0001$ \\
\hline
\end{tabular}

Values expressed as mean \pm SD; Analysis done by student's unpaired $t$ test. The $\mathrm{p}$ values less than 0.05 were considered statistically significant. TP: Total Power, LFnu: Low Frequency component expressed as normalized unit, HFnu: High Frequency component expressed as normalized unit, LF-HF ratio: ratio of low-frequency power to high-frequency power of heart rate variability, Mean RR: Mean RR interval, SDNN: Standard Deviation of Normal to Normal interval, 
Table 3: Comparison of visual evoked potentials between control and diabetic groups.

\begin{tabular}{|c|c|c|c|}
\hline Parameter & Controls $(n=42)$ & Diabetics $(n=44)$ & $\mathrm{p}$ value \\
\hline \multicolumn{4}{|c|}{ Latency of VEP of right eye } \\
\hline N75 (ms) & $69.20 \pm 4.17$ & $69.63 \pm 7.11$ & 0.7361 \\
\hline P100 (ms) & $99.14 \pm 4.23$ & $103.85 \pm 5.91$ & $<0.0001$ \\
\hline N145(ms) & $139.91 \pm 13.68$ & $139.46 \pm 10.80$ & 0.8657 \\
\hline \multicolumn{4}{|c|}{ Amplitude of VEP of right eye } \\
\hline $\mathrm{N} 75-\mathrm{P} 100 \mu \mathrm{v}$ & $6.39 \pm 2.69$ & $5.10 \pm 2.41$ & 0.0222 \\
\hline $\mathrm{P} 100-\mathrm{N} 145 \mu \mathrm{v}$ & $6.26 \pm 2.93$ & $6.49 \pm 3.30$ & 0.7363 \\
\hline \multicolumn{4}{|c|}{ Latency of VEP of left eye } \\
\hline N75 (ms) & $69.19 \pm 4.16$ & $68.99 \pm 6.55$ & 0.8643 \\
\hline P100 (ms) & $99.27 \pm 3.75$ & $103.52 \pm 6.24$ & 0.0003 \\
\hline N145(ms) & $140.62 \pm 11.05$ & $140.12 \pm 10.24$ & 0.8282 \\
\hline \multicolumn{4}{|c|}{ Amplitude of VEP of left eye } \\
\hline N75-P100 $\mu \mathrm{v}$ & $6.06 \pm 2.14$ & $4.56 \pm 2.24$ & 0.0022 \\
\hline $\mathrm{P} 100-\mathrm{N} 145 \mu \mathrm{v}$ & $6.58 \pm 2.97$ & $6.40 \pm 3.36$ & 0.798 \\
\hline
\end{tabular}

Values expressed as mean \pm SD; Analysis done by student's unpaired $t$ test. The $p$ values less than 0.05 were considered statistically significant.

Table 4: Comparison of FBG, insulin and HOMA-IR between control and diabetic groups.

\begin{tabular}{lccc}
\hline Parameter & Controls $(\mathbf{n}=42)$ & Diabetics $(\mathbf{n}=44)$ & p value \\
\hline FBG $(\mathrm{mg} / \mathrm{dl})$ & $80.07 \pm 8.94$ & $153.57 \pm 60.18$ & $<0.0001$ \\
\hline Insulin $(\mu \mathrm{u} / \mathrm{l})$ & $14.53 \pm 5.16$ & $35.26 \pm 19.57$ & $<0.0001$ \\
\hline HOMA IR & $2.89 \pm 1.12$ & $12.23 \pm 5.97$ & $<0.0001$ \\
\hline VEGF $(\mathrm{pg} / \mathrm{ml})$ & $866.45 \pm 182.23$ & $1025.54 \pm 342.67$ & 0.0091 \\
\hline
\end{tabular}

Values expressed as mean $\pm \mathrm{SD}$; Analysis done by student's unpaired $\mathrm{t}$ test. The $\mathrm{p}$ values less than 0.05 were considered statistically significant. FBG: Fasting Blood Glucose, HOMA-IR: Homeostasis Model Assessment of Insulin Resistance. VEGF: Vascular Endothelial Growth Factor.

Table 5: Correlation of VEGF with various parameters of control and diabetic subjects.

\begin{tabular}{lcccc}
\hline \multirow{2}{*}{ Parameters } & \multicolumn{2}{c}{ Controls $(\mathbf{n}=42)$} & \multicolumn{2}{c}{ Diabetics $(\mathbf{n}=44)$} \\
\cline { 2 - 5 } & $\mathbf{r}$ & $\mathbf{p}$ & $\mathbf{r}$ & $\mathbf{p}$ \\
\hline BMI & -0.017 & 0.9146 & 0.1459 & 0.3446 \\
\hline BHR & 0.111 & 0.484 & -0.1314 & 0.9096 \\
\hline DBP & -0.1872 & 0.2353 & -0.0176 & 0.9563 \\
\hline MAP & -0.1731 & 0.2731 & -0.0051 & 0.9734 \\
\hline P100 latency (Rt) & 0.2485 & 0.1125 & 0.3603 & 0.0163 \\
\hline P100 latency (Lt) & 0.2456 & 0.1169 & 0.3693 & 0.0136 \\
\hline FBG & 0.1627 & 0.3034 & -0.0832 & 0.5911 \\
\hline HOMA IR & 0.2175 & 0.1664 & 0.0003 & 0.9981 \\
\hline LF-HF ratio & 0.0869 & 0.5838 & -0.1888 & 0.2198 \\
\hline
\end{tabular}

The $p$ values less than 0.05 were considered statistically significant. BMI: Body Mass Index; BHR: Basal Heart rate, DBP: Diastolic Blood Pressure; MAP: Mean Arterial Pressure, FBG: Fasting Blood Glucose; HOMA IR: Homeostasis Model Assessment of Insulin Resistance.

Table 6: Multiple regression analysis of P100 latency of VEP of right eye (as dependent variable) with VEGF and LF:HF ratio (as independent variables) in diabetic group.

\begin{tabular}{lcccc}
\hline \multirow{2}{*}{$\begin{array}{c}\text { Independent } \\
\text { Variables }\end{array}$} & $\begin{array}{c}\text { Standardized } \\
\text { regression } \\
\text { coefficient beta }\end{array}$ & \multicolumn{2}{c}{$95 \%$ C.I. of B } & p value \\
\cline { 3 - 4 } & 0.36 & 0.001 & 1.011 & 0.016 \\
\hline VEGF & 0.046 & -1.04 & 1.397 & 0.769 \\
\hline LF-HF ratio & bound & $\begin{array}{c}\text { Upper } \\
\text { bound }\end{array}$ & \\
\hline
\end{tabular}

The $\mathrm{p}$ values less than 0.05 were considered statistically significant. VEGF: Vascular endothelial growth factor; LF-HF ratio: ratio of lowfrequency power to high-frequency power of heart rate variability. B: Unstandardized Coefficient; Beta: Standardized Coefficient.

Table 7: Multiple regression analysis of P100 latency of VEP of left eye (as dependent variable) with VEGF and LF:HF ratio (as independent variables) in diabetic group.

\begin{tabular}{lcccc}
\hline & Standardized & \multicolumn{2}{c}{$95 \%$ C.I. of B } & p value \\
\cline { 3 - 4 } $\begin{array}{c}\text { Independen } \\
\text { t Variables }\end{array}$ & $\begin{array}{c}\text { regression } \\
\text { coefficient beta }\end{array}$ & $\begin{array}{c}\text { Lower } \\
\text { bound }\end{array}$ & $\begin{array}{c}\text { Upper } \\
\text { bound }\end{array}$ & \\
\hline VEGF & 0.397 & 0.001 & 1.011 & 0.024 \\
\hline LF:HF & 0.071 & -1.04 & 1.397 & 0.67 \\
\hline
\end{tabular}

The $p$ values less than 0.05 were considered statistically significant. VEGF: Vascular endothelial growth factor; AI: Atherogenic Index; TG/HDL: ratio between triglycerides and high density lipoprotein; LF-HF ratio: ratio of low-frequency power to high-frequency power of 
heart rate variability. B: Unstandardized Coefficient; Beta: Standardized Coefficient.

\section{DISCUSSION}

In the present study, significant increase in diastolic blood pressure in diabetic subjects compared to the age, gender and BMI matched control subjects indicates increased sympathetic activity in these patients as DBP is the reflection of sympathetic vasoconstrictor tone [16]. Further, LF-HF ratio is a sensitive marker of sympathovagal balance $[13,17]$. In the present study significant increase in LF-HF ratio in diabetic group compared to the control group (Table 2) represents considerable Sympathovagal Imbalance (SVI) in diabetic patients. In addition, increase in LF-HF ratio in resting state indicates increased sympathetic activity and decreased vagal tone [17] which was supported by significant increase in LFnu in diabetic group compared to that of the control group. The time-domain indices of HRV (RMSSD, SDNN, NN50, pNN50) were significantly less in diabetic subjects (Table 2), further confirming the decreased vagal tone in these patients, as timedomain indices of HRV represent cardiac parasympathetic drive [14].

The visual evoked potential (VEP), which assesses the integrity of the neural pathways of vision is a very sensitive indicator of electrophysiological changes of visual pathway and even a minor alteration in P100 latency reflects changes in neurophysiological sensitivity in diabetes $[15,18]$. In present study, prolonged latency and decreased amplitude of P100 wave in VEP (Table 3) was observed in diabetics when compared with controls which is similar to other studies $[15,19]$. This delayed latency indicates that there may be pathologic demyelinating lesions of the optic tract although in the present study none had history of optic neuropathy or functional anomalies in peripheral neuropathy [18,20]. P100 latency prolongation has been attributed to retinal or central pathways dysfunction [21].

Angiogenesis/neovascularization plays a key role in developing diabetic retinopathy [22]. VEGF is a highly conserved homodimeric glycoprotein composed of four isoforms resulting from alternative mRNA splicing in humans [23]. VEGF is an angiogenic factor which can be produced by many cells in the eye including retinal pigment epithelium, endothelial cells, pericytes, glial cells, and ganglion cells $[24,25]$. VEGF has been reported to play an important role in mediating the neovascular response in diabetic retinopathy and other ischemic retinal disorders [26]. There was an increased VEGF in diabetics in comparison to controls (Table 4). In the present study the relation between VEGF and probable microangiopathy leading to prolonged latency of P100 is demonstrated by the positive correlation between P100 latency and VEGF in diabetic subjects. The present study is the first of its kind assessing the link between the level of VEGF, which is an indicator of angiogenesis and delay in P100 latency of VEP, a marker of central neuropathy of visual pathway, which is demonstrated by regression analysis (Table 6 and 7). Although significant correlation was present between VEP and VEGF, there was no correlation of LF:HF ratio to VEP and VEGF. The association of autonomic dysfunction i.e, reduced HRV with VEP and VEGF could not be established in the present study which could be probably due to less sample size.

\section{CONCLUSION}

This study demonstrates the presence of SVI in the form of enhanced sympathetic and reduced parasympathetic drive in type 2 diabetes. There is delayed latency of visual evoked potentials and increased vascular endothelial growth factor in type 2 diabetes. The correlation between VEP and VEGF could indicate the probable role of angiogenesis leading to reduced integrity of the neural pathways, responsible for vision seen in the middle age group individuals of diabetes mellitus.

\section{FUTURE PERSPECTIVES}

The other essential risk factors associated with the progression and development of microangiopathy and cardiovascular autonomic neuropathy to be detected in a larger population to treat these patients at an early stage to reduce morbidity and mortality. Studied should be conducted in these individuals to follow non-conventional therapies/life style modifications to see the effect on quality of life of these patients.

\section{CONFLICTS OF INTEREST}

There are no conflicts of interest.

\section{REFERENCES}

1. Surveillance WHOD of ND. Definition, diagnosis and classification of diabetes mellitus and its complications $\llbracket$ : report of a WHO consultation. Part 1, Diagnosis and classification of diabetes mellitus. 1999.

2. Association AD. Diagnosis and Classification of Diabetes Mellitus. Diabetes Care. 2008;31:S55-S60.

3. Whiting DR, Guariguata L, Weil C, Shaw J. IDF diabetes atlas: global estimates of the prevalence of diabetes for 2011 and 2030. Diabetes Res Clin Pract. 2011;94:311-321.

4. Vinik AI, Maser RE, Mitchell BD, Freeman R. Diabetic Autonomic Neuropathy. Diabetes Care. 2003;26:1553-79.

5. Maser RE, Mitchell BD, Vinik AI, Freeman R. The association betweencardiovascular autonomic neuropathy and mortality in individuals withdiabetes: a meta-analysis. Diabetes Care. 2003;6:1895-1901.

6. Maser R, Lenhard M, DeCherney G. Cardiovascular autonomic neuropathy: the clinical significance of its determination. Endocrinologist. 2000;10:27-33.

7. Fong DS, Aiello L, Gardner TW, King GL, Blankenship G, Cavallerano JD, et al. Diabetic Retinopathy. Diabetes Care. 2003;1;26:s99-s102.

8. Klein R, Klein BE, Moss SE, Davis MD, DeMets DL. The Wisconsin Epidemiologic Study of Diabetic Retinopathy. II. Prevalence and risk of diabetic retinopathy when age at diagnosis is less than 30 years. Arch Ophthalmol. 1984;102:520-526.

9. Gayathri V, Vijayalakshmi B, Chandrasekhar M. Electro physiological assessment of neuropathy in visual pathway of diabetes mellitus. J Diabetology. 2012;1:4.

10. Lee IG, Chae SL, Kim JC. Involvement of circulating endothelial progenitor cells and vasculogenic factors in the pathogenesis of diabetic retinopathy. Eye (Lond). 2006;20:546-52. 
11. Mahdy RA, Nada WM, Hadhoud KM, El-Tarhony SA. The role of vascular endothelial growth factor in the progression of diabetic vascular complications. Eye (Lond). 2010;24:1576-84.

12. Aiello LP, Wong JS. Role of vascular endothelial growth factor in diabetic vascular complications. Kidney Int Suppl. 2000;77:113-119.

13. Karthik S, Pal GK, Nanda N, Hamide A, Bobby Z, Amudharaj D, et al. Sympathovagal imbalance in thyroid dysfunctions in females: Correlation with thyroid profi le, heart rate and blood pressure. Indian J Physiol Pharmacol. 2009;53:243-252.

14. Heart rate variability: Standards and measurement, physiological interpretation and clinical use. Task Force of the European Society of Cardiology and the North American Society of Pacing and Electrophysiology. Circulation 1996;93:1043-1065.

15. Heravian J, Ehyaei A, Shoeibi N, Azimi A, Ostadi-Moghaddam H, Yekta AA, et al. Pattern Visual Evoked Potentials in Patients with Type II Diabetes Mellitus. J Ophthalmic Vis Res. 2012;7:225-230.

16. Pal GK, Pal P. Autonomic function tests. In: Textbook of Practical Physiology. 3rd ed. Chennai: Universities Press. 2010;p:282-290.

17. Malliani A. Heart rate variability: from bench to bedside. Eur J Intern Med. 2005;16:12-20.

18. Puvanendran K, Devathasan G, Wong PK. Visual evoked responses in diabetes. J Neurol Neurosurg Psychiatry. 1983;46:643-647.
19. Gupta S, Gupta G, Deshp VK, Visual evoked potentialchanges in patients with diabetes mellitus without retinopathy. Int J Res Med Sci. 2015;3:3591-398.

20. Algan M, Ziegler O, Gehin P, Got I, Raspiller A, Weber M, et al. Visual evoked potentials in diabetic patients. 1989;12:227-229.

21. Mariani E, Moreo G, Colucci GB. Study of visual evoked potentials in diabetics without retinopathy: correlations with clinical findings and polyneuropathy. Acta Neurol Scand. 1990;81:337-340

22. Crawford TN, Alfaro III DV, Kerrison JB, Jablon EP. Diabetic Retinopathy and Angiogenesis. Cur Dia Rev. 2009;5:8-13.

23. Ferrara N, Houck KA, Jakeman LB, Winer J, Leung DW. The vascular endothelial growth factor family of polypeptides. J Cell Biochem. 1991;47:211-218.

24. Aiello LP, Northrup JM, Keyt BA, Takagi H, Iwamoto MA. Hypoxic regulation of vascular endothelial growth factor in retinal cells. Arch Ophthalmol.1995;113:1538-1544.

25. Yang Q, Zwijsen A, Slegers H, Vanden Berghe D. Purification and characterization of VEGF/VPF secreted by human retinal pigment epithelial cells. Endothelium. 1994;2:73-85.

26. Aiello LP, Wong JS. Role of vascular endothelial growth factor in diabeticvascular complications. Kidney International. 2000;58:113-119 